\title{
ARCHIVING OF CURRENT OBSERVATIONAL DATA ON VARIABLE STARS
}

\author{
P. Dubois \\ Centre de Données de Strasbourg \\ Observatoire de Strasbourg \\ 11 rue de l'Université \\ 67000 Strasbourg \\ France
}

\section{WHICH TYPE OF DATA ?}

The first question is : which types of data should we archive for variable stars?

Let us consider the three following types of data: (i) raw data (output of the telescope, time...); (ii) reduced data (flux, magnitude...); (iii) processed data (light curve...).

We generally need the third one and use the reduced data only when we have trouble with the processed data or when we could obtain better processed data. We never quite go back to the raw data. The situation could evolve with raw data obtained with the CCD frame where the reduction procedure is more complex than with electronic counts. Then, at first sight, we have first to archive the processed data, then make an effort on reduced data and raw data.

Processed data. Fortunately, most of these data are published and, in some sense, they are archived. But then the problem is the retrieval of these data and this aspect cannot be separated from the archiving question. Here also, we are lucky, because there are two teams which are collecting the data on variable stars for the astronomical community. One team is in Moscow at the Sternberg Institute and produces the "General Catalog of Variable Stars". The second team is at the Sonneberg Observatory and produced the "Bibliographic Catalog of Variable Stars".

However, all information is not published and much information remains on written cards in both institutes. The other problem is the delay between the appearance of the data in the literature and the publication of the compilation. This is partially filled by the bibliography of SIMBAD (the astronomical database of the CDS). It should be noted that the first version of the GCVS is accessible through SIMBAD and that the accessibility of the $\mathrm{BCVS}$ is also planned.

Reduced data. These data are generally not published and then, unfortunately, often disappear. After a few years, it is difficult and often no more possible, to obtain the reduced data from the authors. We have to make an effort to archive these data. In this field, the amateur astronomer community is organised to save their measurements, and this is also one of the sources of data for the professional astronomers. The most 
complete set of measurements is at the American Association but not all the national associations send their data to it.

For unpublished data, IAU Commission 27 organised also a service in this field : the IAU Archives of Unpublished Observations of Variable Stars (see IBVN3422 for the details). This service is certainly not sufficiently used. It could also be reached at present by electronic mail at the address SIMBAD::IAU27 (on SPAN) or iau27 at simbad.ustrasbg.fr (on INTERNET).

Raw data. The archiving concerns at present mainly the CCD frame for which a general solution should be found. The variable stars data are only a part of the problem. The archiving of the CCD frame seems to be relevant to the observatory where the material is obtained. The archiving of the photographic plates could be linked with the CCD archiving.

\section{RECOMMENDATIONS}

It could be resumed by one sentence : USE THE EXISTING FACILITIES

For practice we could distinguish between the published and unpublished data.

Published data: send reprints to the institutes of Moscow and Sonneberg; send tapes of the data (in case of important amounts of data) to the Data Centers.

Unpublished data: use the IAU Archives of Unpublished Observations of Variable Stars (cf. IBVS No 3422).

Note that these recommendations have a very low cost and distribute the effort of archiving on the overall astronomical community.

\section{Some useful addresses :}

- American Association of Variable Stars Observers (AAVSO)

25, Birch Street, Cambridge, MA 02138-1205, USA

- Association Francaise d'Observateurs d'Etoiles Variables (AFOEV)

Observatoire de Strasbourg, 11 rue de l'Universite, 67000 Strasbourg, France

- Centre de Données de Strasbourg (CDS)

Observatoire de Strasbourg, 11 rue de l'Université, 67000 Strasbourg, France

- iau27@simbad.u-strasbg.fr

- Institute for Astronomy, USSR Academy of Sciences

48, Pjatnitkaya Str., Moscow 109017, USSR

- National Space Science Data Center

NASA, Goddard Space Flight Center, Greenbelt, Maryland 20771, U.S.A.

- Zentralinstitut fur Astrophysik, Sternwarte Sonneberg

Sternwartestrasse 32, O-6400 Sonneberg, Germany 